\title{
Consequências da seletividade alimentar para o estado nutricional na infância: uma
}

\section{revisão narrativa}

\author{
Consequences of food fussiness on nutritional status in childhood: a narrative review \\ Consecuencias de la irritabilidad alimentaria para el estado nutricional en la infancia: una revisíon
} narrativa

Recebido: 30/12/2021 | Revisado: 04/01/2022 |Aceito: 12/01/2022| Publicado: 14/01/2022

\author{
Poliana da Silva Santana \\ ORCID: https://orcid.org/0000-0002-4023-6283 \\ Universidade do Estado da Bahia, Brasil \\ E-mail: pssnutri@outlook.com \\ Thaisy Cristina Honorato Santos Alves \\ ORCID: https://orcid.org/0000-0001-6286-497X \\ Universidade do Estado da Bahia, Brasil \\ E-mail: tcalves@uneb.br
}

\begin{abstract}
Resumo
A seletividade alimentar é um comportamento alimentar comum na infância, caracterizado pela tríade recusa, desinteresse e resistência à alimentação. Esses fatores podem impactar negativamente na ingestão de nutrientes da dieta, contribuindo para alterações do estado nutricional infantil. Dessa forma, o presente estudo buscou evidenciar, mediante uma revisão narrativa de literatura, as consequências do comportamento alimentar seletivo para o estado nutricional de crianças saudáveis. Realizou-se, para tanto, uma busca por estudos originais nas bases de dados Cochrane Library, Embase, MEDLINE/PubMed e Web of Science. Foram identificados 216 estudos sobre a temática; após leitura e remoção de duplicatas, esses resultaram em uma amostra de 10 artigos que compõem o presente trabalho. Através da interpretação, análise e síntese dos achados provenientes do levantamento bibliográfico, pôde-se observar de forma unânime que todos os trabalhos detectaram menor ingestão de frutas e vegetais, assim como de micronutrientes por crianças seletivas. Dos dez estudos analisados, sete demonstraram associação positiva entre o comportamento alimentar seletivo e menores valores de peso, estatura e IMC em crianças. A grande divergência quanto às metodologias empregadas para identificação e classificação da seletividade, bem como, a inexistência - até o momento - de uma definição universalmente aceita de classificação para este comportamento alimentar, são barreiras a serem enfrentadas. Estes são aspectos que tornam mais pesquisas necessárias a fim de possibilitar investigações mais profundas acerca da temática e da associação entre os fatores analisados.
\end{abstract}

Palavras-chave: Seletividade alimentar; Comportamento alimentar; Estado nutricional.

\begin{abstract}
Food fussiness is a frequent eating behavior in childhood, characterized by the triad refusal, disinterest, and resistance to food. These factors can negatively impact the intake of nutrients from the diet, contributing to changes in the nutritional status of children. Thus, this review study sought to emphasize, through a narrative literature review, the consequences of selective eating behavior on the nutritional status of healthy children by searching for original studies in the Cochrane Library, Embase, MEDLINE/PubMed, and Web of Science databases. A total of 216 studies on the subject were identified; after reading and removing duplicates, these resulted in a sample of 10 articles that comprise the present study. Through the interpretation, analysis and synthesis of the findings from the bibliographic survey, all included studies found a lower intake of fruits, vegetables, and micronutrients in selective children. Of the ten studies analyzed, seven indicated a positive association between selective eating behavior and lower weight, height, and BMI in children. Also, some barriers to be faced are the notable divergence in the methodologies used to identify and classify selectivity, and the inexistence - to date - of a universally accepted definition of classification for this eating behavior. These aspects reinforce the need for further research on the theme to enable deeper investigation and understanding of the association between the factors analyzed.
\end{abstract}

Keywords: Food fussiness; Feeding behavior; Nutritional status.

\section{Resumen}

La irritabilidad alimentaria es una conducta alimentaria común en la infancia, caracterizada por la tríada de rechazo, desinterés y resistencia a los alimentos. Estos factores pueden afectar negativamente la ingesta de nutrientes en la dieta, contribuyendo a cambios en el estado nutricional de los niños. Así, a través de la búsqueda de estudios originales en las bases de datos Cochrane Library, Embase, MEDLINE / PubMed y Web of Science, el presente 
estudio buscó resaltar, mediante una revisión narrativa de la literatura, las consecuencias de la conducta alimentaria selectiva para el estado nutricional de niños sanos. Se identificaron 216 estudios sobre el tema; luego de leer y eliminar duplicados, estos dieron como resultado una muestra de 10 artículos que componen el presente trabajo. Mediante la interpretación, análisis y síntesis de los hallazgos de la encuesta bibliográfica, se puede observar unánimemente que todos los estudios detectaron menor ingesta de frutas y verduras, así como de micronutrientes por parte de niños selectivos. De los diez estudios analizados, siete demostraron una asociación positiva entre la conducta alimentaria selectiva y valores más bajos de peso, altura e IMC en los niños. La gran divergencia en cuanto a las metodologías utilizadas para identificar y clasificar la selectividad, así como la inexistencia - hasta ahora - de una definición de clasificación universalmente aceptada para esta conducta alimentaria, son barreras a enfrentar. Estos son aspectosque hacen necesaria una mayor investigación para posibilitar investigaciones más profundas sobre el tema y la asociación entre los factores analizados.

Palabras clave: Irritabilidad alimentaria; Conducta alimentaria; Estado nutricional.

\section{Introdução}

A seletividade alimentar é caracterizada como um comportamento alimentar comumente apresentado durante a infância. São característicos neste quadro recusa, desinteresse e resistência no que se refere à alimentação, tríade que contribui para uma menor diversidade na dieta infantil (Taylor \& Emmett, 2019). Não há consenso sobre uma definição universalmente aceita para seletividade alimentar, ou métodos diagnósticos bem delineados, tendo em vista que esta faz parte de um amplo espectro de comportamentos alimentares comuns na infância (Taylor et al., 2015).

Normalmente, a seletividade alimentar apresenta caráter transitório, porém, em determinados casos pode ser um quadro persistente e perdurar por anos (Taylor et al., 2015), o que eleva as preocupações acerca de suas consequências. Muito especula-se sobre os fatores associados ao desenvolvimento do comportamento alimentar seletivo na infância, e apesar de pesquisas recentes investigarem a perspectiva da seletividade alimentar através de diversas vertentes, suas causas ainda não estão bem elucidadas (Taylor \& Emmett, 2019).

Alguns fatores são descritos como potenciais desencadeadores para o quadro alimentar seletivo: introdução tardia de alimentos mastigáveis no processo de introdução alimentar (Emmett et al., 2018; Taylor \& Emmett, 2019); experiências indesejáveis da alimentação, tais como vômitos, engasgos, refluxos e afins (Levene \& Williams, 2018); e influência parental, expressa através da pressão alimentar (Taylor et al., 2015). Outros fatores que podem contribuir para um quadro maior de seletividade alimentar, são a presença de doenças crônicas, como Diabetes Mellitus tipo 1, e efeitos colaterais de determinados medicamentos, bem como alergias e intolerâncias alimentares (Lam, 2015; Levene \& Williams, 2018).

Sabe-se que uma alimentação balanceada e diversificada, com a presença dos principais grupos alimentares é capaz de fornecer uma ampla variedade de nutrientes, indispensáveis à manutenção da vida e promoção da saúde (Samuel et al., 2018). No caso de crianças seletivas, é comum que menos grupos alimentares se façam presentes na dieta (Taylor \& Emmett, 2019), o que resulta em preocupação com relação ao crescimento e desenvolvimento, tendo em vista que deficiências de micronutrientes estão associadas a agravos à saúde na infância (Pedraza \& Queiroz, 2011).

Impactos negativos ao estado nutricional nesta fase, podem comprometer seriamente o desenvolvimento global infantil e repercutir até a vida adulta (Cunha et al., 2015). Portanto, por se tratar de uma fase em que grande parte do desenvolvimento físico, cognitivo e emocional ocorrem (Cunha et al., 2015), a infância deve ser considerada um momento crucial para avaliação e estímulo de hábitos alimentares saudáveis. Desta forma, o objetivo do presente trabalho é evidenciar a relação e as consequências do comportamento alimentar seletivo para o estado nutricional na infância, mediante uma revisão narrativa da literatura. 


\section{Metodologia}

O presente estudo trata-se de uma revisão narrativa de literatura, uma metodologia descrita por Rother (2007), de caráter qualitativo, cujo objetivo é analisar, interpretar e sintetizar as informações provenientes do levantamento bibliográfico, com a finalidade de atualizar o conhecimento sobre uma temática específica. As fontes de pesquisa consultadas para coleta de dados foram Cochrane Library (CENTRAL), Medical Literature Analysis and Retrieval System Online (MEDLINE/PubMed) e as bases de dados Embase e Web of Science, através do Portal de Periódicos da Coordenação de Aperfeiçoamento de Pessoal de Nível Superior (CAPES).

Para compor o trabalho foram considerados estudos primários e disponíveis na íntegra gratuitamente. Não houve recorte temporal ou preferências quanto ao idioma original do estudo. No entanto, como critérios de exclusão, estudos cujo público-alvo fossem crianças com condições clínicas adversas associadas ao quadro da seletividade alimentar, populações enfermas ou adultos, foram desconsiderados por não se alinharem aos objetivos da pesquisa. Para realização da busca nas bases de dados, foi elaborada uma estratégia de busca utilizando os dois principais descritores em ciências da saúde (DeCS) referentes à temática: "food fussiness" e "nutritional status"; bem como, todos os respectivos sinônimos para ambos.

Os operadores booleanos "OR" e "AND" foram utilizados, para conectar os termos entre si, abrangendo os resultados de pesquisa para os termos oficiais e seus sinônimos, tornando-a mais específica. A triagem dos estudos foi realizada em duas etapas. Na primeira, houve a leitura superficial - título e resumo. Subsequentemente, a leitura integral dos estudos previamente filtrados, confirmou se os critérios de elegibilidade explicitados foram devidamente cumpridos, bem como, o alinhamento entre os estudos encontrados e os objetivos do presente trabalho.

\section{Resultados}

A busca inicial nas bases de dados identificou 216 estudos acerca da temática, que após leitura e remoção de duplicatas, resultaram em uma amostra de 10 artigos que compõem o presente trabalho. Foram identificados três tópicos encontrados de forma recorrente nos estudos selecionados, a respeito dos quais discorreremos a seguir: avaliação e classificação da seletividade alimentar; ingestão dietética; e relação entre seletividade alimentar e estado nutricional.

\section{Avaliação e classificação da seletividade alimentar}

Para abordar a relação entre seletividade alimentar e estado nutricional, cabe inicialmente verificar os métodos utilizados pelos estudos para identificar e classificar esse comportamento alimentar. Mostrou-se bem evidente que existem variados parâmetros para avaliar e classificar seletividade alimentar na infância, não havendo, ainda, uma padronização para tal classificação. Assim, a ferramenta a ser utilizada em pesquisa variou entre os estudos, e tal pluralidade de possibilidades tende a dificultar comparações entre estes.

Em todos os estudos, a avaliação e classificação da seletividade alimentar infantil foi realizada através da aplicação de questionários autoadministráveis com os responsáveis pelas crianças. Um questionário amplamente utilizado foi o Child Eating Behaviour Questionnaire (CEBQ), em sua versão integral, ou apenas a subescala referente à agitação alimentar, a qual é capaz de aferir seletividade e neofobia alimentares. O Child Feedind Questionnaire (CFQ) também foi utilizado em algumas pesquisas, sendo capaz de averiguar a seletividade alimentar, através do padrão alimentar infantil. Em ambos os questionários, as respostas variam em uma escala Likert de 1 a 5 - "nunca" a "sempre" - onde quanto maior a pontuação final, maior a associação com o comportamento analisado.

O estudo de Brown e Perrin (2020) foi o que utilizou mais ferramentas para a classificação. Esse estudo compilou sete medidas distintas para avaliação do comportamento alimentar seletivo: subescala de agitação alimentar de seis itens do CEBQ; três itens do CFQ; Stanford Feeding Questionnaire (oito itens); Eating Behaviour Questionnaire (três itens); sete itens do 
Inventário de Comportamento Alimentar Infantil do Oregon Research Institute; Escala de Neofobia Alimentar Infantil (seis itens) e um Questionário de Alimentação Seletiva (dez itens).

Utilizando perguntas provenientes de estudos anteriores para avaliar a seletividade alimentar, através da frequência de determinados comportamentos alimentares, Kwon et al. (2017) selecionaram quatro comportamentos alimentares específicos para a classificação em sua amostra. Comer pequenas quantidades, comportamento neofóbico, recusa em comer grupos de alimentos específicos e preferência por um método específico de preparo dos alimentos foram os tópicos utilizados como direcionamentos para a condução das perguntas. Logo, crianças com pontuação $>3$ foram classificadas como seletivas.

Dubois et al. (2007) utilizaram um questionário proveniente do Estudo Longitudinal de Pais e Filhos da Avon (ALSPAC), para avaliar em três períodos, dois comportamentos alimentares infantis distintos: a seletividade e o comer excessivo. As crianças foram classificadas como seletivas através de escores obtidos para cada pergunta, quando "sempre" faziam uma refeição diferente da comida de outros membros da família, "frequentemente" se recusavam a comer a comida certa e aquelas que "frequentemente" se recusavam a comer. Um total de 0 pontos indica a ausência do comportamento, já a soma de 3 pontos indica a presença do comportamento.

Já o estudo conduzido em Taiwan por Chao (2018), utilizou uma versão adaptada de um questionário de preferências e comportamentos alimentares, baseada em uma pesquisa do Departamento de Saúde do Reino Unido. Concomitantemente, utilizou-se uma versão adaptada do CEBQ para os hábitos alimentares da população em questão, classificando as crianças como seletivas ou não e compreendendo o consumo e evitação alimentar por grupos alimentares.

Digno de nota que a utilização de escalas de comportamento alimentar sem a apresentação de uma conceituação explícita sobre alimentação seletiva, tende a ser mais conveniente por não induzir uma resposta ou inferir juízo de valor aos responsáveis. Dessa forma, se torna possível analisar o comportamento alimentar mediante as experiências alimentares interpretadas pelos pesquisadores, através das respostas fornecidas pelos cuidadores; não através de uma expectativa subjetiva, do ponto de vista pessoal dos cuidadores. Entretanto, cabe salientar a importância da padronização dos métodos para avaliação da seletividade entre os pesquisadores dessa área específica.

\section{Seletividade alimentar e ingestão dietética}

Crianças classificadas como seletivas no estudo de Brown et al. (2018) obtiveram pontuação geral inferior na análise do consumo de frutas inteiras, vegetais totais, verduras, feijão e alimentos proteicos totais. Ao investigar os principais alimentos recusados por sua amostra, Xue et al. (2015) chegaram à conclusão de que, as crianças seletivas possuem maior rejeição a vegetais $(51,1 \%)$, soja $(27,9 \%)$, carnes $(25,7 \%)$, ovos $(21,7 \%)$, cereais $(19,4 \%)$, leite $(13,0 \%)$, alimentos doces $(11,1 \%)$ e frutas $(9,8 \%)$.

Já Kwon et al. (2017), identificaram em seu estudo os grupos de alimentos mais aceitos e mais preteridos pelo grupo seletivo. Sendo estes: mariscos, feijão e vegetais mais recusados; enquanto peixes, frutas e ovos foram os alimentos mais aceitos. É interessante destacar que normalmente as crianças exigiam uma forma de preparo específica para os frutos do mar e feijão.

No estudo de Galloway et al. (2005), o consumo médio diário para frutas e vegetais pelo grupo seletivo foi identificado abaixo do valor de referência (sete porções diárias), estipuladas pelo Guia Alimentar Americano; assim como a ingestão de grãos e carnes. Entre as crianças seletivas, o percentual de consumo alimentar que atendia às orientações da Pirâmide do Guia Alimentar foi menor. Apenas $2 \%$ e $0 \%$ atenderam às recomendações para porções de frutas e vegetais, respectivamente.

O estudo conduzido por Chao (2018) identificou uma menor aceitação alimentar por parte das crianças seletivas: $16,7 \%$ das crianças recusavam alimentos de forma geral, porém, especialmente frutas e verduras; enquanto 14,8\% substituíam 
as refeições principais por doces ou salgadinhos. Entre esse público, também foi elevada a rejeição para carnes $(37,1 \%)$, vegetais $(38,9 \%)$, frutas $(22,2 \%)$ e tipos específicos de vegetais ou frutas $(21,6 \%)$.

Li e colaboradores (2017) avaliaram as categorias de alimentos evitados por crianças seletivas em três grupos de faixa etária distintos: 6 a 11 meses; 12 a 23 meses; e 24 a 35 meses. O primeiro apresentou rejeição para os grupos de leite e laticínios $(27,8 \%)$, sobremesas $(20,4 \%)$, feijão e produtos de feijão $(16,7 \%)$ e carnes $(16,7 \%)$. Já as demais faixas etárias apresentaram rejeição a grupos semelhantes: vegetais (12-23 meses: 33\%; 24-35 meses: 50,8\%) e grãos e produtos derivados (12-23 meses: 17\%; 24-35 meses: 10,7\%). A particularidade entre esses grupos ficou por conta da rejeição a ovos (15,1\%) na faixa de 12-23 meses; e carnes $(23,2 \%)$ para a faixa de $24-35$ meses.

O estudo evidencia ainda que o consumo médio de ovos e frutas foi inferior nas crianças classificadas como seletivas e que evitavam estes alimentos. No entanto, curiosamente, as crianças classificadas como seletivas e que evitavam vegetais ou carnes, acabaram tendo consumo energético maior associado a esses alimentos, do que crianças não seletivas (Li et al., 2017).

Os estudos de Antoniou et al. (2016), Galloway et al. (2005) e Kwon et al. (2017) apresentaram semelhanças ao apontar que crianças seletivas possuem menor consumo energético, quando comparadas a crianças não seletivas. No entanto, as diferenças encontradas foram pouco significativas. Galloway et al. (2005) destacaram que, apesar da redução, o consumo de energia e macronutrientes estava dentro das recomendações. Porém, Kwon et al. (2017) realçaram que crianças seletivas e que tendem a consumir quantidades menores de alimentos, possuem ingestão energética significativamente menor quando comparadas àquelas não seletivas. Diferindo destes achados, o estudo conduzido por Xue et al. (2015) identificaram menor ingestão de energia, proteína e carboidratos no grupo de alimentação seletiva.

Apenas os estudos de Galloway et al. (2005) e Xue et al. (2015), analisaram a ingestão de fibras pelos grupos, e em ambos os estudos foi detectado baixo consumo de fibras nos grupos de alimentação seletiva. No entanto, Galloway et al. (2005) chamam atenção para o fato de que, apesar do grupo seletivo consumir menor quantidade de fibras, ambos os grupos consumiram fibras abaixo da recomendação dietética. Xue et al. (2015) destacam o fato de que a baixa ingestão de fibras é um reflexo direto da ingestão limitada de vegetais pelas crianças com comportamento seletivo.

Quanto aos micronutrientes, Galloway et al. (2005) relatam em seu estudo o consumo inadequado de vitamina E, cálcio e magnésio para todas as crianças. No entanto, havia o risco aumentado de crianças mais seletivas não conseguirem alcançar as recomendações para as vitaminas C e E. Menor ingestão de vitaminas A e B6, tiamina, riboflavina, e vitamina C foi identificada em crianças seletivas na faixa de 6-11 meses no estudo de Li et al. (2017). Porém, ainda assim, a ingestão média de vitamina A e riboflavina se encontravam acima dos valores de referência de ingestão adequada. No estudo de Xue e colaboradores (2015) foi detectada ingestão significativamente reduzida de vitaminas A, C e E, tiamina, riboflavina; e dos minerais cálcio, magnésio, ferro, zinco e cobre, em crianças seletivas em comparação ao grupo não seletivo. Já o estudo conduzido por Brown e colaboradores (2018) não identificou ingestões reduzidas para micronutrientes na comparação entre as amostras.

\section{Seletividade alimentar e estado nutricional}

Foi possível identificar que a maioria dos estudos convergiu ao apontar associações positivas entre seletividade alimentar e estado nutricional na infância, sendo relatados com unanimidade entre estes, valores médios inferiores encontrados nos índices antropométricos referentes a peso, estatura e IMC para idade.

Antoniou et al. (2016), identificaram diferenças na estatura e nos valores de IMC encontrados, sendo estes menores em crianças seletivas. O estudo aponta ainda, que crianças seletivas são mais propensas a apresentar baixo peso e menores taxas de sobrepeso na faixa etária de cinco anos. Similarmente, Galloway et al. (2005), encontraram menor IMC e percentual de gordura corporal significativamente mais baixo no grupo de alimentação seletiva. 
Dubois et al. (2007) relatam que grande proporção da amostra com comportamento alimentar seletivo, apresentou baixo peso (IMC inferior ao $10^{\circ}$ percentil), ao passo que uma pequena proporção apresentou risco de sobrepeso (IMC entre os percentis $85^{\circ}$ e $\left.94,9^{\circ}\right)$; diferentemente do grupo não seletivo. Através de análises multivariadas, o estudo ainda conseguiu estabelecer que, ser classificado como seletivo entre os 2,5 e 4,5 anos aumenta as chances de estar abaixo do peso aos 4,5 anos em até 2,4 vezes.

Chao (2018) identificou medidas de peso, altura e IMC médios inferiores na população seletiva do estudo; bem como peso para idade, altura para idade e IMC para idade significativamente mais baixos nesta população. Comparando os grupos, observou-se também que apenas os valores médios para o grupo seletivo se encontravam abaixo da mediana para a população. A incidência de valores abaixo do percentil 15 para os índices peso para idade, altura para idade e IMC para idade foi maior nesse grupo.

Kwon et al. (2017) identificaram que crianças seletivas que consumiam pequenas quantidades, apresentaram menores pontuações escore-z de peso para idade e IMC para idade. Para a faixa etária entre 4 e 5 anos, o índice de altura para idade também foi menor. Ao passo que, aqueles que apresentavam maior recusa de grupos alimentares específicos obtiveram menor valor encontrado de estatura para a idade na mesma faixa etária. $\mathrm{O}$ estudo estabelece ainda, que a associação é mais forte em crianças mais velhas, sendo que estas apresentam alturas mais curtas e IMC mais baixos do que aquelas na faixa etária mais jovem.

Roy et al. (2020), em um estudo conduzido com alunos de uma escola primária, encontraram associação significativa entre magreza e seletividade alimentar, medida através das escalas de comportamento alimentar do CEBQ. Enquanto Xue et al. (2015) identificaram, através de análises de regressão linear bruta e ajustada, medidas antropométricas significativamente mais baixas entre as crianças seletivas, sendo estas: peso, altura e IMC. A análise do escore-z também identificou reduções para os indicadores de altura para idade, peso para idade e IMC para idade no grupo seletivo.

O estudo de Xue et al. (2015), analisou ainda a relação entre os grupos alimentares mais evitados pelas crianças seletivas, e a redução nos valores de z-escore. Os achados indicam que crianças com rejeição acentuada para vegetais apresentam redução na altura para a idade e no peso para a idade; a rejeição às carnes foi associada a redução na altura para idade; peso para idade e IMC para a idade; da mesma forma, aqueles que rejeitavam ovos apresentaram alterações nas três medidas escore-z analisadas.

Apenas os estudos de Brown et al. (2018), Brown e Perrin (2020) e Li et al. (2017), não encontraram associação entre seletividade alimentar e estado nutricional, sendo os três estudos transversais. Brown et al. (2018) e Brown e Perrin (2020) avaliaram a relação entre seletividade alimentar e alguns índices para avaliação do estado nutricional (escore-z para peso, altura, IMC e peso para altura) em sua população. O estudo mais recente aponta ainda, que não há relação entre os dados antropométricos analisados e nenhum dos componentes comumente associados à alimentação seletiva. Enquanto Li et al. (2017), ao utilizarem peso e altura em sua análise, relataram distribuições de peso e altura semelhantes para crianças seletivas e não seletivas.

\section{Discussão}

Hodiernamente, os comportamentos alimentares na infância vêm ganhando destaque e tornando-se motivo de preocupação entre pais e responsáveis durante o processo de alimentação infantil. Como reflexo disto, a atenção no meio científico se encontra concentrado especialmente nas duas últimas décadas. Uma rápida busca nas bases de dados, comprova o crescente olhar voltado para estabelecer a relação entre os comportamentos alimentares e estado nutricional na infância, bem como, as consequências desta relação. No entanto, quando o assunto é o comportamento alimentar seletivo, o foco é relativamente novo. 
Conforme descrito na metodologia deste trabalho, em um corpo amostral relativamente significativo, poucos estudos avaliaram a relação e os impactos da seletividade para o estado nutricional de crianças saudáveis. Diante de tal situação, tratase de um comportamento difícil de ser avaliado objetivamente, não havendo ainda um padrão-ouro para identificar e classificar as crianças dentro do amplo espectro de comportamentos alimentares comuns nesta fase. O que existem são observações comportamentais pertinentes ao quadro, bem como questionários validados para avaliar - através do olhar dos cuidadores - a alimentação seleta.

Levene e Williams (2018), destacam a importância de o profissional da área da saúde saber diferenciar apropriadamente o comportamento alimentar seletivo de condições clínicas adversas, como o Transtorno Alimentar Restritivo Evitativo (TARE). Apesar da seletividade estar presente na caracterização do quadro de TARE, esta possui critérios diagnósticos bem delineados, e repercussões clínicas à saúde bem definidas; diferentemente do comportamento alimentar seletivo. No entanto, como a terminologia já sugere, comportamentos alimentares são comuns na infância, ao passo que transtornos alimentares não.

Por isto, a partir da suspeita do quadro, é importante que a análise minuciosa de todos os aspectos relacionados ao estado nutricional infantil seja realizada, para verificar possíveis comprometimentos associados a alimentação mais seletiva. Dessa forma, torna-se possível avaliar o nível de gravidade, visando distinguir se a seletividade está dentro de um espectro de normalidade, ou, apresenta prognóstico favorável para desenvolvimento de distúrbios do crescimento, desenvolvimento e deficiências de micronutrientes (Emmett et al., 2018).

Uma dificuldade encontrada na comparação entre os estudos, foi fruto da divergência quanto às definições adotadas e metodologias empregadas na análise do comportamento seletivo infantil. $\mathrm{O}$ fato de alguns estudos apresentarem uma definição para alimentação seletiva aos responsáveis, pode induzir tais cuidadores a relatar os comportamentos de forma mais acentuada do que realmente o são; enquanto outros estudos, apenas direcionaram as perguntas e analisaram a pontuação para determinar a presença da seletividade, sem apresentar uma conceituação do quadro aos cuidadores.

Todos os estudos analisados englobaram os cuidadores principais (mãe, pai ou ambos), na avaliação e classificação do quadro de seletividade, tendo em vista que os questionários sobre os comportamentos e preferências alimentares das crianças eram respondidos por estes. No entanto, por temer repercussões ao estado nutricional e saúde global da criança, estes podem subestimar o peso corporal e estado nutricional em crianças classificadas como seletivas (Li et al., 2017).

A qualidade da dieta se torna uma das grandes preocupações dos cuidadores de crianças seletivas. No entanto, Leung et al. (2012) destacam que, o interesse alimentar e apetite não são constantes durante a infância, podendo haver uma diminuição fisiológica do apetite, especialmente na fase da primeira infância, o que, segundo estudo publicado por Lam (2015), não tende a causar alterações significativas na ingestão calórica ou nutricional diárias.

De todos os estudos que se propuseram a analisar a ingestão de micronutrientes pela amostra seletiva, apenas um não encontrou ingestões inferiores na comparação com o grupo não seletivo. Há confluência nos demais estudos, quanto a detecção da ingestão inferior de algumas vitaminas (A, C, E, B6, tiamina e riboflavina), e minerais (cálcio, zinco, magnésio e cobre) pelo grupo de alimentação seletiva. O que pode, muito provavelmente, estar associado ao fato de que comumente, menos grupos alimentares se fazem presentes na dieta habitual de crianças seletivas (Taylor \& Emmett, 2019).

Pedraza e Queiroz (2011), ressaltam a relação entre deficiências de micronutrientes e efeitos deletérios na infância, como o aumento das taxas de morbimortalidade, comprometimento potencial do crescimento físico, entre outros agravos à saúde. Sendo os nutrientes que se destacam nesse contexto: ferro, zinco e vitamina A, que possuem relação com limitações ao crescimento e desenvolvimento infantis por sua presença em vias metabólicas importantes. As crianças seletivas apresentaram menor consumo dos nutrientes citados. 
A suplementação pode ser uma alternativa nesses casos. No entanto, esta é adotada quando a composição e qualidade da dieta são questionáveis e podem acarretar prejuízos, para além do estado nutricional, ao crescimento e desenvolvimento infantis (Lam, 2015; Leung et al., 2012). Ainda assim, esta possui caráter temporário, auxiliando no processo de mudanças comportamentais e dietéticas a serem implementadas (Emmett et al., 2018), tendo em vista que, jamais deve-se substituir a ingestão de alimentos saudáveis por suplementos alimentares (Leung et al., 2012).

Mostra-se evidente que para avaliar até que ponto a ingestão reduzida de micronutrientes pode impactar na saúde e estado nutricional infantis, mais estudos longitudinais são necessários. Um estudo de acompanhamento dessas crianças, pode fornecer um novo panorama quanto à extensão das possíveis consequências a longo prazo em um quadro mais persistente.

$\mathrm{O}$ veredito encontrado sobre a relação entre comportamento alimentar seletivo e estado nutricional se mostrou misto na literatura selecionada. Dos dez estudos que compõem o corpo amostral, sete associaram positivamente os fatores; porém, três não encontraram relação significativa. Dos três estudos cujo resultado diferem da maioria, dois são conduzidos pela mesma pesquisadora (Brown et al., 2018; Brown \& Perrin, 2020). No terceiro estudo foram utilizados apenas peso e altura para realizar a comparação entre os grupos (Li et al., 2017).

Apesar de resultados divergentes em alguns estudos, de forma geral, todos aqueles que realizaram a análise da ingestão por grupos alimentares concordam no tocante à rejeição de vegetais e frutas recorrentemente entre crianças seletivas. Taylor et al., (2016) encontraram resultados semelhantes, com o adicional de que essas crianças tendem a consumir mais alimentos ultraprocessados, fato que não foi explorado em detalhes pelos estudos analisados.

Os achados encontrados na presente revisão, confirmam a preocupação para com o estado nutricional de crianças seletivas, tendo em vista que uma alimentação equilibrada e diversificada é essencial para promoção de saúde e bem-estar aos indivíduos, sendo esta alcançada através da presença de grupos alimentares diversos no consumo diário, para proporcionar a gama de nutrientes indispensáveis ao ser humano, como vitaminas, minerais, carboidratos, lipídeos, proteínas, fibras e diversos compostos bioativos (Samuel et al., 2018).

Ao se deparar com um quadro de seletividade alimentar infantil, é necessário que o profissional esteja atento e apto para identificar o potencial do comportamento apresentado, visando a possibilidade de prejuízos futuros relacionados ao bemestar geral e estado nutricional. Dessa forma, torna-se mais fácil a tomada de decisões em prol de uma intervenção eficaz, haja vista que muitas das crianças descritas como seletivas por seus cuidadores, podem não apresentar comportamentos alimentares fora do comum para a faixa etária (Levene \& Williams, 2018).

A análise da composição alimentar da dieta, identificando a presença e frequência dos principais grupos alimentares, exames bioquímicos, recordatório alimentar, dados antropométricos, bem como a aplicação de questionários validados em estudos específicos sobre a temática - como os que compõem os estudos analisados - podem auxiliar o profissional a visualizar o quadro como um todo (Levene \& Williams, 2018).

A importância de identificar e intervir o mais precocemente possível no comportamento alimentar seletivo, está atrelado a maiores chances de impedir a persistência do quadro e seus possíveis danos a médio e longo prazo, como o desenvolvimento de transtornos alimentares na adolescência (Taylor \& Emmett, 2019). As estratégias para prevenir ou melhorar a alimentação seletiva, devem ser pensadas de forma individualizada através da composição geral do quadro e trabalhadas com a criança e seus cuidadores.

Assim, a dinâmica familiar como um todo, se mostrou um objeto de observação presente na maioria dos estudos. Por isso, trabalhar expectativas mais realistas e condizentes com as necessidades alimentares infantis (tamanho e quantidade das porções); bem como, analisar as tabelas de classificação dos índices antropométricos de referência juntamente aos responsáveis, pode tranquilizá-los quando o comportamento apresentado não representar potenciais riscos à saúde e/ou estado nutricional. 


\section{Considerações Finais}

Os resultados encontrados na literatura acerca da relação entre seletividade alimentar e estado nutricional na infância, se mostraram muito relevantes, podendo contribuir para a atuação na prática clínica. Estes podem auxiliar o profissional na visualização do quadro, contribuindo assim, para intervenções mais eficazes quando houver a identificação de riscos potenciais ao crescimento, desenvolvimento e estado nutricional infantis. No entanto, mais estudos devem ser estimulados sobre a inferência da seletividade alimentar no estado nutricional da criança, em especial por meio de estudos longitudinais.

Cabe salientar que para que os estudos se tornem comparáveis entre si de forma mais homogênea, o estabelecimento de definições e métodos diagnósticos padrões se faz necessário. Por fím, como sugestão para estudos futuros referentes à temática, a ampliação da análise da investigação dos fatores envolvidos no desenvolvimento desse comportamento alimentar, podem contribuir para uma melhor compreensão de tal condição e para o estabelecimento de um prognóstico mais efetivo quanto às repercussões clínicas à saúde e ao desenvolvimento infantis. Ademais, novos achados poderão viabilizar intervenções gerais e específicas voltadas para o quadro da seletividade alimentar infantil.

\section{Referências}

Antoniou, E. E., Roefs, A., Kremers, S. P. J., Jansen, A., Gubbels, J. S., Sleddens, E. F. C., \& Thijs, C. (2016). Picky eating and child weight status development: a longitudinal study. Journal of Human Nutrition and Dietetics, 29(3), 298-307. https://doi.org/10.1111/jhn.12322

Brown, C. L., Perrin, E. M., Peterson, K. E., Herb, H. E. B., Horodynski, M. A., Contreras, D., \& Lumeng, J. C. (2018). Association of picky eating with weight status and dietary quality among low-income preschoolers. Academic pediatrics, 18(3), 334-341. https://doi.org/10.1016/j.acap.2017.08.014

Brown, C. L., \& Perrin, E. M. (2020). Defining picky eating and its relationship to feeding behaviors and weight status. Journal of behavioral medicine, 43(4), 587-595. https://doi.org/10.1007/s10865-019-00081-w

Chao, H. C. (2018). Association of picky eating with growth, nutritional status, development, physical activity, and health in preschool children. Frontiers in pediatrics, 6, 22. https://doi.org/10.3389/fped.2018.00022

Cunha, A. J. L. A. D., Leite, Á. J. M., \& Almeida, I. S. D. (2015). Atuação do pediatra nos primeiros mil dias da criança: a busca pela nutrição e desenvolvimento saudáveis ‘ł. Jornal de Pediatria, 91, S44-S51. https://doi.org/10.1016/j.jped.2015.07.002

Dubois, L., Farmer, A., Girard, M., Peterson, K., \& Tatone-Tokuda, F. (2007). Problem eating behaviors related to social factors and body weight in preschool children: A longitudinal study. International Journal of Behavioral Nutrition and Physical Activity, 4(1), 1-10. https://doi.org/10.1186/1479-5868-4-9

Emmett, P. M., Hays, N. P., \& Taylor, C. M. (2018). Antecedents of picky eating behaviour in young children. Appetite, 130, 163-173. https://doi.org/10.1016/j.appet.2018.07.032

Galloway, A. T., Fiorito, L., Lee, Y., \& Birch, L. L. (2005). Parental pressure, dietary patterns, and weight status among girls who are "picky eaters". Journal of the American Dietetic Association, 105(4), 541-548. https://doi.org/10.1016/j.jada.2005.01.029

Kwon, K. M., Shim, J. E., Kang, M., \& Paik, H. Y. (2017). Association between picky eating behaviors and nutritional status in early childhood: Performance of a picky eating behavior questionnaire. Nutrients, 9(5), 463. https://doi.org/10.3390/nu9050463

Lam, J. (2015). Picky eating in children. Frontiers in pediatrics, 3, 41. https://doi.org/10.3389/fped.2015.00041

Leung, A. K., Marchand, V., Sauve, R. S., Canadian Paediatric Society, \& Nutrition and Gastroenterology Committee. (2012). The 'picky eater': The toddler or preschooler who does not eat. Paediatrics \& child health, 17(8), 455-457. https://doi.org/10.1093/pch/17.8.455

Levene, I. R., \& Williams, A. (2018). Fifteen-minute consultation: The healthy child: "My child is a fussy eater!". Archives of Disease in ChildhoodEducation and Practice, 103(2), 71-78. http://dx.doi.org/10.1136/archdischild-2016-311787

Li, Z., van der Horst, K., Edelson-Fries, L. R., Yu, K., You, L., Zhang, Y., \& Wang, J. (2017). Perceptions of food intake and weight status among parents of picky eating infants and toddlers in China: A cross-sectional study. Appetite, 108, 456-463. https://doi.org/10.1016/j.appet.2016.11.009

Pedraza, D. F., \& de Queiroz, D. (2011). Micronutrientes no crescimento e desenvolvimento infantil. Journal of Human Growth and Development, 21(1), 156171. https://doi.org/10.7322/jhgd.20005

Rother, E. T. (2007). Revisão sistemática X revisão narrativa. Acta Paulista de Enfermagem, 20(2), v-vi. https://doi.org/10.1590/S0103-21002007000200001

Roy, S., Bandyopadhyay, S., Bandyopadhyay, L., Dasgupta, A., Paul, B., \& Mandal, S. (2020). Nutritional status and eating behavior of children: A study among primary school children in a rural area of West Bengal. Journal of family medicine and primary care, 9(2), 844. 10.4103/jfmpc.jfmpc_965_19

Samuel, T. M., Musa-Veloso, K., Ho, M., Venditti, C., \& Shahkhalili-Dulloo, Y. (2018). A narrative review of childhood picky eating and its relationship to food intakes, nutritional status, and growth. Nutrients, 10(12), 1992. https://doi.org/10.3390/nu10121992 
Research, Society and Development, v. 11, n. 1, e52511125248, 2022

(CC BY 4.0) | ISSN 2525-3409 | DOI: http://dx.doi.org/10.33448/rsd-v11i1.25248

Taylor, C. M., Wernimont, S. M., Northstone, K., \& Emmett, P. M. (2015). Picky/fussy eating in children: Review of definitions, assessment, prevalence and dietary intakes. Appetite, 95, 349-359. https://doi.org/10.1016/j.appet.2015.07.026

Taylor, C. M., Northstone, K., Wernimont, S. M., \& Emmett, P. M. (2016). Macro-and micronutrient intakes in picky eaters: a cause for concern?. The American journal of clinical nutrition, 104(6), 1647-1656. https://doi.org/10.3945/ajen.116.137356

Taylor, C. M., \& Emmett, P. M. (2019). Picky eating in children: Causes and consequences. Proceedings of the Nutrition Society, 78(2), 161-169. https://doi.org/10.1017/S0029665118002586

Xue, Y., Lee, E., Ning, K., Zheng, Y., Ma, D., Gao, H., \& Zhang, Y. (2015). Prevalence of picky eating behaviour in Chinese school-age children and associations with anthropometric parameters and intelligence quotient. A cross-sectional study. Appetite, 91, 248-255. https://doi.org/10.1016/j.appet.2015.04.065 